\title{
The efficacy of Enhanced Recovery after Surgery (ERAS) for elderly patients with intertrochanteric fractures who received surgery: study protocol for a randomized, blinded, controlled trial
}

\author{
Mengchen Yin®, Yinjie Yan, Zhaoxiang Fan, Niankang Fang, Hongbo Wan, Wen Mo* and Xuequn Wu*
}

\begin{abstract}
Background: Intertrochanteric fracture (ITF) is increasing with the rapid increase in the aging population, often causes a high mortality rate in old patients and increases the economic burden of the family and society. ERAS (Enhanced Recovery after Surgery) is a powerful guarantee for patients to accelerate their recovery after surgery. TCM (traditional Chinese medicine) promotes repair of injured tissues and eliminates traumatic aseptic inflammation. Therefore, this prospective randomized controlled clinical trial aims to evaluate the clinical effect of the evidence-based ERAS pathway of integrating TCM with western medicine on perioperative outcomes in ITF patients undergoing intramedullary fixation and provide reliable evidence-based data for applying the program to clinical practice.
\end{abstract}

Methods/design: We will conduct a prospective randomized, blinded, controlled trial to compare the effectiveness of ERAS care pathway with traditional care pathway and to investigate whether the ERAS care pathway can improve the perioperative outcome in ITF patients undergoing intramedullary fixation. A total of 60 patients with ITF will be enrolled and treated with the two care pathway, respectively. Length of stay, economic indicators, Harris score, VAS score, time to get out of bed, 30-day readmission rates, postoperative transfusion rates, discharge to home, and mortality will be evaluated. Any signs of acute adverse reactions will be recorded at each visit during treatment.

Discussion: Although an evidence-based process using the best available literature and Delphi expert-opinion method has been used to establish an ERAS pathway of integrating TCM with western medicine, there is a lack of consensus about its effectiveness. This trial will provide convincing evidence about the effect of ERAS pathway.

Trial registration: Registered on 12 October 2019. Trial number is ChiCTR1900026487.

Keywords: Intertrochanteric fracture, Enhanced recovery after surgery, Perioperative period, Integrating TCM with and western medicine, Randomized controlled trial

\footnotetext{
* Correspondence: mw2218@126.com; wxqlzjj@163.com

Department of Orthopaedics, LongHua Hospital, Shanghai University of Traditional Chinese Medicine, Shanghai, China
}

(c) The Author(s). 2020 Open Access This article is distributed under the terms of the Creative Commons Attribution 4.0 International License (http://creativecommons.org/licenses/by/4.0/), which permits unrestricted use, distribution, and reproduction in any medium, provided you give appropriate credit to the original author(s) and the source, provide a link to the Creative Commons license, and indicate if changes were made. The Creative Commons Public Domain Dedication waiver (http://creativecommons.org/publicdomain/zero/1.0/) applies to the data made available in this article, unless otherwise stated. 


\section{Introduction}

With the aging of society, the incidence of intertrochanteric fracture (ITF) is increasing. It is easy to bring bedsore, urinary tract infection, lung infection, and other complications. Intertrochanteric fracture has become a major public health issue with a high mortality rate for old patients and increased the economic burden $[1,2]$. Currently, ITF is treated with both surgical and non-surgical methods. Surgical treatment has great advantages in alleviating pain, restoring hip function, improving quality of life, and avoiding complications such as deep vein thrombosis and cardiovascular accident caused by long-term bed rest, so surgical treatment is the first choice of treatment [3-6]. Currently, PFNA (proximal femoral nail anti-rotation, PFNA) is a mature surgical treatment for senile ITF, which is characterized by maintaining strong fixation, biomechanical stability, and minimal invasion [7, 8].

ERAS (Enhanced Recovery after Surgery) is one of the two important development directions leading the development of modern surgery in the twenty-first century, which is first proposed by Kehlet in 1997 [9]. ERAS aims to integrate the perioperative diagnosis and treatment concept by optimizing a series of effective approaches and methods with evidence-based medical evidence, so as to reduce trauma invasion and alleviate the stress reaction caused by surgery. The core of the ERAS is a powerful guarantee for patients to accelerate their recovery after surgery [10-13]. At present, ERAS has been applied in the treatment of many orthopedic diseases including artificial joint replacement [14].

Currently, major efforts are being conducted to transfer the lessons learned from other surgical specialties and incorporate ERAS protocols into postoperative care of patients undergoing hip surgery. ERAS protocols for artificial joint replacement have been described that it can reduce postoperative mortality and increase satisfaction for patients. However, to our best knowledge, ERAS programs for patients with ITF have not been described. Furthermore, traditional Chinese medicine including acupuncture, manipulative therapy, and oral Chinese medicine can improve blood circulation in local tissues, relax tense and spasmodic muscles, promote repair of injured tissues, and eliminate traumatic aseptic inflammation. Therefore, it is of great significance to establish an effective plan and management pathway integrated Chinese and western medicine to accelerate postoperative recovery of ITF. With the objective in mind, we have conducted a systematic literature search to guarantee the comprehensiveness of the study; Delphi expert-opinion method was used to establish the ERAS pathway. Here, ERAS programs consist of a multidisciplinary evidence-based approach to preoperative management, intraoperative control, and rehabilitation for ITF, and preoperative multidisciplinary collaboration had been already established.

Therefore, this prospective, randomized, controlled clinical trial aims to evaluate the clinical effect of an evidence-based ERAS pathway integrated Chinese and western medicine on perioperative outcomes in ITF patients undergoing intramedullary fixation and provide reliable evidence-based data.

\section{Materials and methods Study design}

This study is a prospective, outcome assessor- and data analyst-blinded, randomized controlled design study that compared an ERAS care pathway cohort with standardized care pathway cohort. Emergency admissions with a primary diagnosis of ITF between January 2020 and July 2021 in our hospital will be selectively enrolled. All patients will receive their operation at our hospital where elective surgeries take place predominantly, and they will be treated with PFNA intramedullary fixation at our institution.

The objective of this proposed study is to investigate whether ERAS care pathway will lead to perioperative improvement in ITF patients undergoing intramedullary fixation. The principal investigator (PI) is responsible for the overall project and organizing Steering Committee meetings. PIs of sub-center departments are responsible for gathering experts to carry out the project. An independent Steering Committee will be responsible for affairs such as participants' safety, meetings, recruitment and follow-up of participants, and quality control. The coordinating center is responsible for communicating protocol modifications and providing materials. This trial includes a 2-week treatment period and a 3-month follow-up period. Outcome assessments will be conducted at baseline, as well as at 3, 14, 30, and 90 days (Fig. 1).

\section{Eligibility criteria Inclusion criteria}

Participants who meet the criteria below are eligible [15]:

- The patients are over 75 years old.

- X-ray or CT indicates unstable ITF.

- Treated with PFNA intramedullary fixation.

- Being willing to undergo surgery with the ERAS pathway.

- Being willing to give informed consent.

\section{Exclusion criteria}

The exclusion criteria are as follows [15]: 


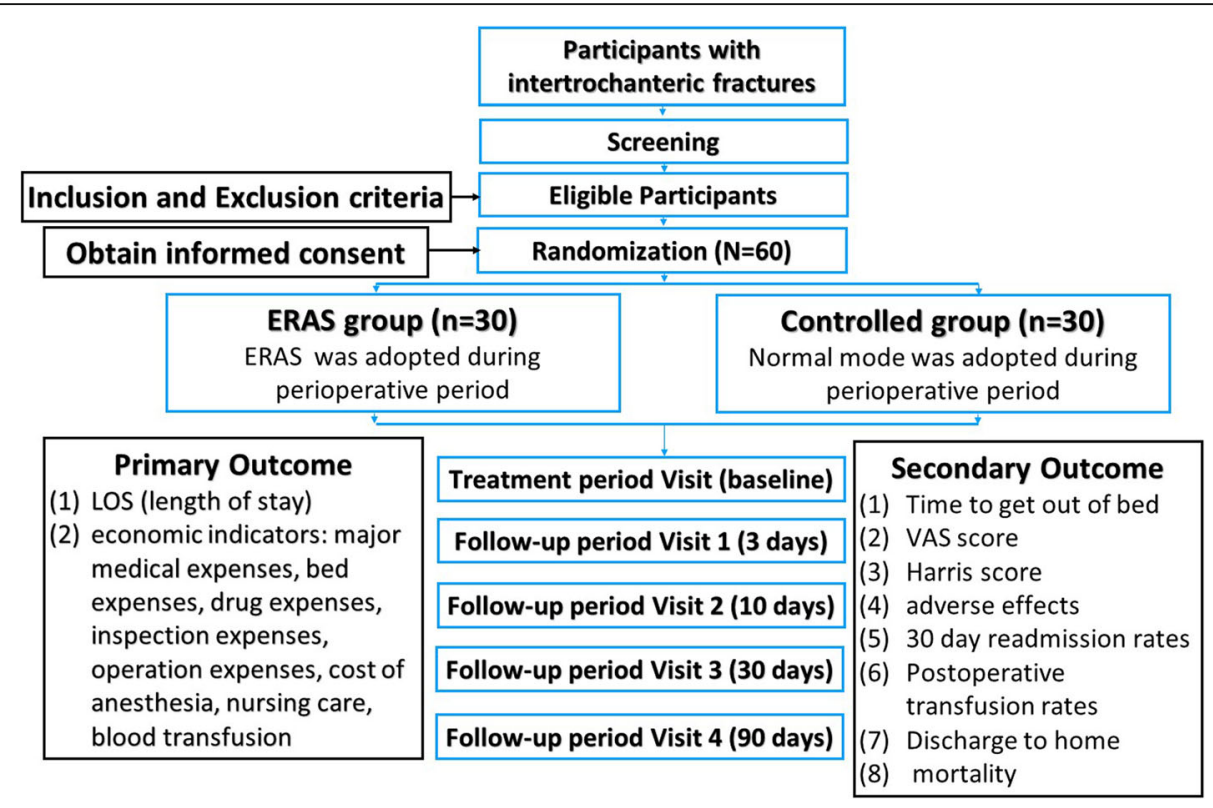

Fig. 1 Participant processing and the schedule of evaluation

- Patients are with open fractures or pathological fracture caused by tumor, infection, or tuberculosis.

- Patients are with congenital hip dysplasia or osteonecrosis of the femoral head.

- Hip surgery before enrollment.

- Failure to understand or sign informed consent.

\section{Patient population and recruitment procedure}

Participants will be recruited from Longhua Hospital affiliated to Shanghai University of Traditional Chinese Medicine. Prospective participants will be interviewed by the coordinators and informed of the eligibility criteria and the procedure. Those who are eligible and willing to participate in the study will be screened initially by baseline assessment and then diagnosed based upon clinical manifestations, physical examination, and imaging. Participants will be informed that participating in the trial was absolutely voluntary and withdrawal from the trial can be made at any time. In case of withdrawal, the data collected will not be deleted and will be used in the final analyses. A data compilation form including all variables of interest and all potential risks will be completed by the corresponding research center. The information obtained will be stored in an electronic database for subsequent statistical analysis. Recruitment will start in January 2020 and is expected to end in July 2021. The final follow-up of all participants will be completed on 30 September 2021. The overview of the participant processing and the schedule of evaluation is provided in Fig. 1.
Patient and public involvement

Patients and/or the public will not be involved in the study design and study enrolment.

Ethics

This study is to be conducted in accordance with the principles of the Declaration of Helsinki and has been approved by Sichuan Regional Ethics Review Committee on Traditional Chinese Medicine (TCM) [16]. The trials have been approved by the appropriate Institutional Review Boards. The clinical trial has been approved by the Institutional Review Board of Longhua Hospital, Shanghai University of TCM. All participants will be given sufficient time to reach a decision to sign the consent form prior to the study in compliance with Good Clinical Practice Guidelines that guides the appropriate use of TCM in clinical practice. Then, the participants will be randomized into two groups of different treatments. Reporting will be guided by the CONSORT statement $[17,18]$.

\section{Intervention}

\section{ERAS care pathway}

An evidence-based process using the best available literature and Delphi expert-opinion method was used to establish the ERAS pathway integrated Chinese and western medicine. The basic components of the multidisciplinary ERAS pathway which we used are shown in Table 1. The principles include educational program, management of nutrition, management of dietary, management of sleep, management of pain selection of 
Table 1 The basic components of the multi-disciplinary ERAS pathway

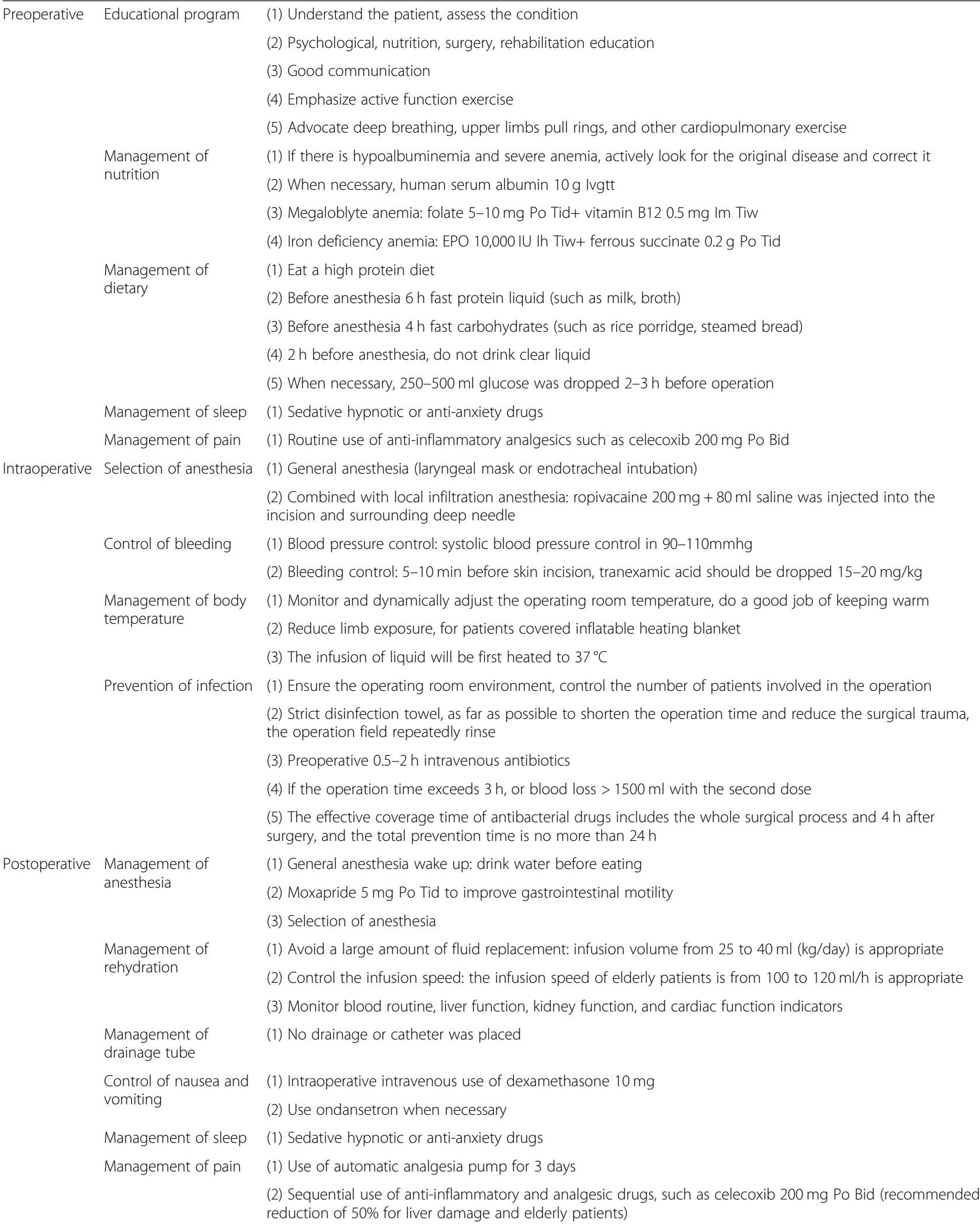

(2) Combined with local infiltration anesthesia: ropivacaine $200 \mathrm{mg}+80 \mathrm{ml}$ saline was injected into the incision and surrounding deep needle

Control of bleeding (1) Blood pressure control: systolic blood pressure control in 90-110mmhg

(2) Bleeding control: 5-10 min before skin incision, tranexamic acid should be dropped 15-20 mg/kg

Management of body (1) Monitor and dynamically adjust the operating room temperature, do a good job of keeping warm temperature

(2) Reduce limb exposure, for patients covered inflatable heating blanket

(3) The infusion of liquid will be first heated to $37^{\circ} \mathrm{C}$

Prevention of infection (1) Ensure the operating room environment, control the number of patients involved in the operation (2) Strict disinfection towel, as far as possible to shorten the operation time and reduce the surgical trauma, the operation field repeatedly rinse

(3) Preoperative $0.5-2 \mathrm{~h}$ intravenous antibiotics

(4) If the operation time exceeds $3 \mathrm{~h}$, or blood loss $>1500 \mathrm{ml}$ with the second dose

(5) The effective coverage time of antibacterial drugs includes the whole surgical process and $4 \mathrm{~h}$ after surgery, and the total prevention time is no more than $24 \mathrm{~h}$

Postoperative Management of anesthesia

(1) General anesthesia wake up: drink water before eating

(2) Moxapride $5 \mathrm{mg}$ Po Tid to improve gastrointestinal motility

(3) Selection of anesthesia

Management of rehydration

Management of drainage tube

(1) Avoid a large amount of fluid replacement: infusion volume from 25 to $40 \mathrm{ml}$ (kg/day) is appropriate

(2) Control the infusion speed: the infusion speed of elderly patients is from 100 to $120 \mathrm{ml} / \mathrm{h}$ is appropriate

(3) Monitor blood routine, liver function, kidney function, and cardiac function indicators

(1) No drainage or catheter was placed

Control of nausea and

(1) Intraoperative intravenous use of dexamethasone $10 \mathrm{mg}$

vomiting

(2) Use ondansetron when necessary

Management of sleep

(1) Sedative hypnotic or anti-anxiety drugs

Management of pain

(1) Use of automatic analgesia pump for 3 days

(2) Sequential use of anti-inflammatory and analgesic drugs, such as celecoxib $200 \mathrm{mg}$ Po Bid (recommended reduction of $50 \%$ for liver damage and elderly patients)

Management of activity
(1) Emphasis on early hip, knee, and ankle active flexion and extension function exercise, to increase muscle strength 
Table 1 The basic components of the multi-disciplinary ERAS pathway (Continued)

\author{
(2) Exercise passive joint flexion and extension of hip, knee, and ankle joints with the help of the physician \\ and CPM, at least three times a day, at least 15 min each time \\ (3) Asked frequently turn over, clap back \\ (4) Acupuncture \\ (5) Manipulation \\ (6) Oral traditional Chinese medicine
}

anesthesia, control of bleeding, management of body temperature, prevention of infection, management of anesthesia, management of rehydration management of drainage tube, control of nausea and vomiting, and management of activity.

\section{Traditional care pathway}

There was no detailed plan in the controlled group, and anti-infection, anti-coagulation, and other measures were taken to prevent complications during the perioperative period.

\section{Randomization and allocation}

After the screening, patients will be randomized into two groups with an allocation ratio of 1:1. The randomization will be generated via SAS PROC PLAN software (SAS Inc., Cary, NC, USA) by an independent third-party clinical research organization (Institute of Basic Research in Clinical Medicine, China Academy of Chinese Medical Science) and concealed from the researchers by a senior data manager who is not involved in the study. The group assignment list will be sealed in opaque envelopes and be opened by the researchers following informed consent procedures and baseline testing.

\section{Blinding}

All the investigators, physicians, nurses, assessors, analysts, and participants will be blinded to the group assignment until the end of the trial, when all statistical analyses are finished. If, after the first administration, any adverse event potentially related to the treatment occurs, the study physician will re-evaluate the participant, and PI will decide whether the non-blinded procedure is necessary. If non-blinding is required, the allocation information will be provided.

\section{Outcome measurements}

\section{Primary outcome measurement}

LOS (length of stay) associated with ITF is a major public health issue due to the aging population, and it is the most objective outcome of evaluating the recovery pathway. Furthermore, high LOS would correspond with increases in postoperative complications $[14,19]$. So we choose the LOS at discharge as the primary outcome measurement to assess the speed of recovery. Economic indicators including major medical expenses, bed expenses, drug expenses, inspection expenses, operation expenses, cost of anesthesia, nursing care, and blood transfusion are the other primary outcome measurement.

\section{Secondary outcome measurements}

Postoperative joint-specific function will be measured using the Harris score, which is widely used to assess the joint function of life for ITF patients. The Harris score is composed of 10 questions, 2 questions (ROM and absence of deformity) for the physician physical examination component and 8 questions for the patientreported outcome component [20-24]. VAS is a reliable and valid measurement of pain. It has a horizontal, 100mm-long line, with "no pain" recorded on the left side (score 0) and "pain as bad as it could be" on the right side (score 10) [25]. The other secondary outcomes included time to get out of bed, 30-day readmission rates, postoperative transfusion rates, discharge to home, and mortality.

\section{Safety assessments}

Infection, deep venous thrombosis, and cardiovascular accidents will be recorded at each visit during treatment.

\section{Sample size calculation}

We calculate the sample size according to our primary study. We conducted a preliminary clinical trial and pilot trial about ERAS care pathway versus traditional care pathway from January 2018 to May 2018. The primary outcome parameter was the LOS. Based on the previous results, we found that the primary outcome parameter of the ERAS care pathway group was 5.8 days and that of the traditional care pathway group was 9.2 days. According to the formula of the rate in completely random design, $n_{1}=$ $n_{2}=\frac{\left[u_{a / 2} \sqrt{2} \bar{p}(1-\bar{p})+u_{\beta} \sqrt{p_{1}\left(1-p_{1}\right)}+p_{2}\left(1-p_{2}\right)\right]}{\left(p_{1}-p_{2}\right)^{2}}$, among which $n_{1}$ and $n_{2}$ were the number of patients in the two $u_{a / 2}=1.96$ when type 1 error is 0.05 and $u_{\beta}=1.282$ when type II error is 0.1 , respectively, in two-sided tests. $\bar{p}$ is the mean of $p_{2}$ [26]. A two-sided 5\% significance level and $90 \%$ power in detecting treatment differences were considered, and the above relevant data were input into SPSS 
20.0 software. This number of patients actually provided less than $80 \%$ power, considering an estimated dropout rate of $20 \%$. Therefore, we will recruit a total of 72 patients with 36 patients in each group.

\section{Statistical analyses}

Prior to all analyses, a detailed statistical analysis protocol will be developed. All data will be analyzed in the clinical research center of Longhua Hospital affiliated to Shanghai University of TCM by statisticians blinded to allocation using the SPSS 20.0 statistical software (SPSS Inc., Chicago, IL). Efficacy and safety analyses will be conducted according to the intention-to-treat principle using the "last observation carried forward" rule. Before randomization, baseline characteristics will be collected as descriptive statistics for each patient, including gender, age, BMI, duration of symptoms, preoperative red blood cell count, and preoperative hemoglobin count. The data analysis of the primary outcome is based on the per-protocol population as a supportive analysis. Mean, standard deviation, median, quartiles and interquartiles for continuous variables, and frequency for categorical variables will be calculated. Continuous variable followed the normal distribution will be presented as means with standard deviations (SDs) and calculated by an independent sample Student's $t$ test; otherwise, the data will be expressed as medians with ranges, and nonparametric tests will be used. Categorical variables will be expressed as number (\%) and analyzed by $\chi^{2}$ test or Fisher's exact test. A $P$ value of less than 0.05 is defined as statistically significant with two-sided $90 \%$ confidence intervals (CIs). Missing data will be input with the last observed response carried forward for all measures using the "last value carried forward" principle.

\section{Quality control}

Prior to the clinical trial, we will carry out unified training to make sure all the physicians, nurses, and assessors involved fully understand the process of the trial, including selecting patient screening and selection, case report form writing, and manipulation details. To guarantee the quality of the whole trial, rigorous monitoring will be performed by three trained quality supervisors. During the trial, supervisors will check on case report forms and intervention twice a month. After verifying the case report forms, data will be input into the database by two full-time research members independently. The standard operating procedures (SOP) will be invariably followed. Dropouts, withdrawals (and the reasons), and any compliance of all patients occurring will be recorded in detail by the inspectors throughout the treatment and followup period.

\section{Discussion}

The surgical stress response associated with major surgery describes fundamental metabolic changes that lead to increased catabolism, immunosuppression, free radical production, and hypercoagulable states. These physiologic alterations have been linked to changes in organ function resulting in undesirable postoperative morbidity, complications, pain, fatigue, and extended convalescence. ERAS attempts to decrease the surgical stress response to minimize postoperative complications and improve surgical outcomes and functional rehabilitation after major surgery.

ITF commonly occurred in the elderly and caused high mortality rate due to the complications of loss of walking ability. Due to the great importance of good functional outcome and avoidance of serious postoperative complications, the general consensus is that early surgery is the first choice of treatment for the elderly patients and PFNA is a mature surgical procedure.

Several clinical literatures describing ERAS on arthroplasty have been published previously in the literature, but there is a lack of prospective high-quality data from larger cohorts on it for improved recovery after surgery. In particular, we are unaware of published ERAS program on the field of ITF. Based on the theory of TCM, manipulations, acupuncture, and oral Chinese medicine are of great significance in promoting fracture healing and improving patients' whole body state. With the objective, we have conducted a systematic literature search including Wan Fang Data, CNKI databases, Vip Journal Integration Platform, Chinese BioMedical databases, PubMed, MEDLINE, EMBASE, Cochrane Library, and ISI web of knowledge, and ERAS pathway integrated Chinese and western medicine was established by Delphi expert-opinion process including ten experts. The new ERAS project is consist of a multidisciplinary evidencebased approach to preoperative management, intraoperative control, and rehabilitation.

To our best knowledge, our study is the first elaborately designed, randomized, controlled trial to evaluate the clinical effect of the ERAS project integrated Chinese and western medicine on perioperative outcomes in ITF patients undergoing PFNA. A hallmark of ERAS is coordination between care services before and after surgery and continual evaluation of postoperative course with attention toward pain control, functional recovery, and patient satisfaction to improve standards of care. So the study is designed as a comprehensive study of LOS, economic indicators, pain relief, functional outcome, and complications. Outcome measurements are widely used in research to establish baselines, evaluate the effect of an intervention, and motivate patients' self-evaluation. LOS was the most commonly assessed metric in comparative analyses. And a comparative reduction in LOS 
was reported in most studies using the ERAS protocol. Improved cost-effectiveness of surgical interventions is also a primary objective when implementing strategies for faster recovery in this study. The calculation of costeffectiveness provided is based on data provided by our Department of Finance. The study is also to evaluate whether ERAS program can lead to a reduction in hospitalization costs, care costs, and nursing costs, which supports the cost-effectiveness of "fast-track" protocols in PFNA surgery. The VAS measurements, which have been found valid, reliable, and easy to apply in researches, are often used as the criterion standard to evaluate the pain intensity. The Harris score, a selfadministered questionnaire, has been widely adopted as the criterion standard to estimate disease activity for its reliability and validity. Another aim of the study lies in reducing both intra- and postoperative adverse events and complication, which have the potential to impair the patients' perioperative well-being and to prolong recovery. Especially in elderly patients, or in those with severe comorbidities, strict patient selection is key to both eligibility for safe ERAS and treatment in specialized shortstay clinics that may not have an ICU available. Administration of prophylactic medication against infections and thrombosis, prevention of hypothermia, and fluid imbalance, as well as operative measures, has therefore become integral to successful implementation of ERAS [27-30].

The lack of good quality RCTs in the field leaves us with notable gaps in our knowledge, and in clinical practice, many decisions have to be taken without the benefit of high-quality evidence. So we decide to conduct a prospective, randomized, controlled clinical trial, which also ensures the compliance of participants and meets ethical considerations, to closely detect the clinical efficacy of the ERAS program. The present study is built on our preliminary open experiment: a small sample-sized, randomized, and controlled trial of the ERAS program for patients with ITF. The result of the preliminary trial has shown that LOS of the ERAS care pathway group was 5.8 days and that of the traditional care pathway group was 9.2 days.

We hope that this trial, with a larger number of patients, can provide adequate statistical power to further analyze and explore the efficacy of ERAS. Thus, quality control is vital to the whole study, as we described in the protocol. To perform a reliable study, we will carry out unified training to make sure all the physicians, nurses, and assessors involved in the trials fully understand the process and details of ERAS program before the clinical trial. Hopefully, this trial will produce high-quality evidence pertaining to the efficacy and safety of the ERAS in treating patients with ITF. The results will aid in clinical decision-making for the management and provide useful information that can be incorporated into future guidelines.

\section{Abbreviations \\ ERAS: Enhanced Recovery after Surgery; ITF: Intertrochanteric fracture: LOS: Length of stay; PFNA: Proximal femoral nail anti-rotation; \\ TCM: Traditional Chinese medicine}

\section{Acknowledgements \\ We thank all the medical staff in the department of orthopedics and traumatology of Longhua Hospital affiliated to Shanghai University of Traditional Chinese Medicine for their meticulous care of patients who have given us great help. We also thank the patients for their support and trust, and their medical treatment is also a great help to us.}

\section{Authors' contributions}

YMC, YYJ, and FZX are co-first authors of this manuscript, contributing equally to the design, conduct of the trials, and drafting of the manuscript. All authors participated in the design of the study and performed the trial. MW and WXQ are co-corresponding authors of this manuscript, contributing equally and supervised and coordinated the clinical trial. All authors read and approved the final manuscript.

\section{Funding}

This work was sponsored by research grants from the Research Project of Shanghai Science and Technology Commission (201940231).

\section{Availability of data and materials}

The datasets used and/or analyzed during the current study are available from the corresponding author on reasonable request. Ethics approval and consent to participate. The study design, procedures, and informed consent procedure were approved by the Longhua Hospital. Consent to participate will be obtained from the participants.

\section{Ethics approval and consent to participate}

This study is to be conducted in accordance with the principles of the Declaration of Helsinki and has been approved by the Sichuan Regional Ethics Review Committee on Traditional Chinese Medicine. Details of the trial program have been approved by the appropriate Institutional Review Boards.

\section{Consent for publication}

Not applicable.

\section{Competing interests}

The authors declare that they have no competing interests.

Received: 5 January 2020 Accepted: 10 February 2020

Published online: 05 March 2020

\section{References}

1. Bhowmick K, Matthai T, Boopalan PRJ, Jepegnanam TS. Decision making in the management of malunion and nonunion of intertrochanteric fractures of the hip. Hip Int. 2019;1120700019863410:1-8.

2. Cho SH. Outcomes of dynamic hip screw augmented with trochanteric wiring for treatment of unstable type A2 intertrochanteric femur fractures. Injury. 2019;50(3):816.

3. Jung EY, Oh IT, Shim SY, Yoon BH, Sung YB. The effect of valgus reduction on the position of the blade of the proximal femoral nail antirotation in intertrochanteric hip fractures. Clin Orthop Surg. 2019;11(1):36-42.

4. Imerci A, Aydogan NH, Tosun K. The effect on outcomes of the application of circumferential cerclage cable following intramedullary nailing in reverse intertrochanteric femoral fractures. Eur J Orthop Surg Traumatol. 2019;29(4): 835-42.

5. Han YH, Jeong HJ, Sohn MH, Yoon SJ, Lim ST. Incidence and severity of femoral head avascularity after femoral neck or intertrochanteric fractures on preoperative bone single photon emission computed tomography/ computed tomography: preliminary study. Nucl Med Commun. 2019;40(3): 199-205.

6. Li J, Zhang $L$, Zhang $H$, et al. Effect of reduction quality on post-operative outcomes in 31-A2 intertrochanteric fractures following intramedullary 
fixation: a retrospective study based on computerised tomography findings. Int Orthop. 2019;43(8):1951-9.

7. Makki D, Matar HE, Jacob N, Lipscombe S, Gudena R. Comparison of the reconstruction trochanteric antigrade nail (TAN) with the proximal femoral nail antirotation (PFNA) in the management of reverse oblique intertrochanteric hip fractures. Injury. 2015;46(12):2389-93.

8. Nishiura T, Nozawa M, Morio $H$. The new technique of precise insertion of lag screw in an operative treatment of trochanteric femoral fractures with a short intramedullary nail. Injury. 2009;40(10):1077-83.

9. Kehlet H. Multimodal approach to control postoperative pathophysiology and rehabilitation. Br J Anaesth. 1997;78(5):606-17.

10. Ljungqvist O, Scott M, Fearon KC, et al. JAMA Surg. 2017;152(3):292-8.

11. Grant MC, Yang D, Wu CL, Makary MA, Wick EC. Impact of enhanced recovery after surgery and fast track surgery pathways on healthcareassociated infections: results from a systematic review and meta-analysis. Ann Surg. 2017;265(1):68-79.

12. Malczak P, Pisarska M, Piotr M, Wysocki M, Budzynski A, Pedziwiatr M. Enhanced recovery after bariatric surgery: systematic review and metaanalysis. Obes Surg. 2017;27(1):226-35.

13. Pearson KL, Hall NJ. What is the role of enhanced recovery after surgery in children? A scoping review. Pediatr Surg Int. 2017;33(1):43-51.

14. Zhu S, Qian W, Jiang C, Ye C, Chen X. Enhanced recovery after surgery for hip and knee arthroplasty: a systematic review and meta-analysis. Postgrad Med J. 2017:93(1106):736-42.

15. Kang $\mathrm{Y}$, Liu J, Chen $\mathrm{H}$, et al. Enhanced recovery after surgery (ERAS) in elective intertrochanteric fracture patients result in reduced length of hospital stay (LOS) without compromising functional outcome. J Orthop Surg Res. 2019;14(1):209.

16. Fuson RL, Sherman M, Van Vleet J, Wendt T. The conduct of orthopaedic clinical trials. J Bone Joint Surg Am. 1997;79(7):1089-98.

17. Begg C, Cho M, Eastwood S, et al. Improving the quality of reporting of randomized controlled trials. The CONSORT statement. JAMA. 1996;276(8):637-9.

18. Schulz KF, Altman DG, Moher D. CONSORT 2010 statement: updated guidelines for reporting parallel group randomized trials. Ann Intern Med. 2010;152(11):726-32.

19. Dietz N, Sharma M, Adams S, et al. Enhanced recovery after surgery (ERAS) for spine surgery: a systematic review. World Neurosurg. 2019;130:415-26.

20. Bagheri Z, Jafari $P$, Faghih $M$, Allahyari $E$, Dehesh T. Testing measurement equivalence of the SF-36 questionnaire across patients on hemodialysis and healthy people. Int Urol Nephrol. 2015;47(12):2013-21.

21. Jenkinson C, Peto V, Coulter A. Making sense of ambiguity: evaluation in internal reliability and face validity of the SF 36 questionnaire in women presenting with menorrhagia. Qual Health Care. 1996;5(1):9-12.

22. Kili S, Wright I, Jones RS. Change in Harris hip score in patients on the waiting list for total hip replacement. Ann R Coll Surg Engl. 2003;85(4):269-71.

23. Mahomed NN, Arndt DC, McGrory BJ, Harris WH. The Harris hip score: comparison of patient self-report with surgeon assessment. J Arthroplast. 2001;16(5):575-80.

24. Soderman P, Malchau $\mathrm{H}$. Is the Harris hip score system useful to study the outcome of total hip replacement? Clin Orthop Relat Res. 2001;384:189-97.

25. Campbell WI, Lewis S. Visual analogue measurement of pain. Ulster Med J. 1990;59(2):149-54

26. Fayers P. Approaches to sample size estimation in the design of clinical trials--a review. By A. Donner, Statistics in Medicine, 3, 199-214 (1984). Stat Med. 1993;12(17):1643.

27. Hoeksma HL, Van Den Ende CH, Ronday HK, Heering A, Breedveld FC. Comparison of the responsiveness of the Harris Hip Score with generic measures for hip function in osteoarthritis of the hip. Ann Rheum Dis. 2003;62(10):935-8.

28. Hung $M$, Hon SD, Cheng $C$, et al. Psychometric evaluation of the lower extremity computerized adaptive test, the modified Harris hip score, and the hip outcome score. Orthop J Sports Med. 2014;2(12):2325967114562191.

29. Kalairajah Y, Azurza K, Hulme C, Molloy S, Drabu KJ. Health outcome measures in the evaluation of total hip arthroplasties--a comparison between the Harris hip score and the Oxford hip score. J Arthroplast. 2005;20(8):1037-41.

30. Li Z, Wang Q, Li B, Bai B, Zhao Q. Influence of enhanced recovery after surgery programs on laparoscopy-assisted gastrectomy for gastric cancer: a systematic review and meta-analysis of randomized control trials. World J Surg Oncol. 2017;15(1):207.

\section{Publisher's Note}

Springer Nature remains neutral with regard to jurisdictional claims in published maps and institutional affiliations.

\section{Ready to submit your research? Choose BMC and benefit from:}

- fast, convenient online submission

- thorough peer review by experienced researchers in your field

- rapid publication on acceptance

- support for research data, including large and complex data types

- gold Open Access which fosters wider collaboration and increased citations

- maximum visibility for your research: over $100 \mathrm{M}$ website views per year

At BMC, research is always in progress.

Learn more biomedcentral.com/submissions 